\title{
Raumrelevante Probleme der Entwicklung von Großwohnsiedlungen in den neuen Bundesländern
}

\begin{abstract}
Kurzfassung
Nahezu jeder vierte Bürger der neuen Bundesländer lebt derzeit noch (!) in einer der über 300 Großsiedlungen mit jeweils 1000 und mehr Wohnungen. Umfangreiche bautechnische Sanierungsprogramme und Maßnahmen zur Wohnumfeldverbesserung haben in den vergangenen Jahren das Ziel verfolgt, diese Großsiedlungen als unverzichtbare Elemente der Wohnungsversorgung sozial $\mathrm{zu}$ stabilisieren und städtebaulich weiterzuentwickeln. Dennoch geben wachsene Fluktuationsraten, eine vielfach steigende potentielle Migrationsbereitschaft und partielle Leerstände insbesondere in wirtschaftlich strukturschwächeren Räumen ernste Hinweise darauf, in Zukunft neben den generellen Entwicklungsanforderungen der Gebiete den spezifischen raumrelevanten Problemen, d.h. der Differenziertheit ihrer räumlichen Einordnung, mehr Aufmerksamkeit zu widmen. Erfahrungen, wie sie seit den 70er Jahren mit unterschiedlichen Interventionsmodellen in französischen „Grands Ensembles” gesammelt werden, können dabei prinzipiell auch für Großsiedlungen in den neuen Bundesländern interessant sein.
\end{abstract}

\section{Forschungen am Institut für Regionalentwicklung und Strukturplanung (IRS) zu Großsiedlungsstrategien}

Seit seiner Neugründung im Januar 1992 stellt das Institut für Regionalentwicklung und Strukturplanung e. V. (IRS) in Erkner bei Berlin einen nicht unerheblichen Teil seiner Forschungskapazität in den Dienst anwendungsbezogener Grundlagenforschung zur Perspektive der Großwohnsiedlungen bzw. großen Neubaugebiete, die im Zeitraum der 50er bis 80er Jahre in der ehemaligen DDR entstanden sind.

Nahezu jeder vierte Bürger der neuen Bundesländer lebt derzeit in einem dieser 386 Neubaugebiete mit jeweils 1000 und mehr Wohnungen.' Wenn auch von vielen vor der Wende nicht geliebt, so doch akzeptiert als ,Inseln der Gegenwart", sind solche Großsiedlungen wie Berlin-Marzahn, -Hellersdorf und -Hohenschönhausen, Leipzig-Grünau, Dresden-Gorbitz, Magdeburg-Olvenstedt und viele andere inzwischen als „Platten-Ghettos", „Gegenwelten der Zivilisation"2 oder „Orte - nicht zum Leben" ${ }^{3}$ massiv ins öffentliche Gerede gekommen, was ihre Stigmatisierung als künftige Notstandsgebiete und soziale Brennpunkte verstärkt.

Demgegenüber setzt die am IRS betriebene Forschung, ausgehend von einer umfangreichen Bestandsaufnahme, die 1990/91 noch am damaligen Institut für Städtebau und Architektur in Ostberlin durchgeführt worden war, ${ }^{4}$ von Anfang an auf eine vor allem bestandsorientierte Stabilisierung und Weiterentwicklung dieser Siedlungen mit dem Ziel der Schaffung eigenständiger und integrierter, funktionstüchtiger und vielfältig strukturierter Stadtteile als ,normale" Stadtgebiete mit sozial und demographisch gemischten Bevölkerungsstrukturen, einer ökonomisch und ökologisch sinnvollen Nutzungsmischung und einem lebendigen Miteinander von Erhalten und Verändern, Weiterbauen und Emeuern.
1992 arbeitete das IRS am Konzept eines neuen spezifischen Forschungsfeldes im Rahmen des Ressortforschungsprogramms Experimenteller Wohnungs- und Städtebau (ExWoSt) mit. ${ }^{5}$ Innerhalb der in dieses Feld einbezogenen elf Modellvorhaben betreute das IRS als Forschungsträger 1992 bis 1994 die Arbeiten zum Modellbeispiel Ludwigsfelde, Land Brandenburg. ${ }^{6}$

Seit 1994/95 nimmt das IRS die Forschungsbegleitung für das Bund-Länder-Förderungsprogramm ,Städtebauliche Weiterentwicklung großer Neubaugebiete in den neuen Länderm und Berlin-Ost" wahr, in das inzwischen über 150 Großwohnsiedlungen in mehr als 120 Städten einbezogen sind. Auf dem im September 1996 in Leipzig-Grünau durchgeführten Fachkongre $B$ ist eine $Z$ wischenbilanz der bisherigen Fördermaßnahmen insbesondere bei der Gestaltung des Wohnumfeldes der Plattenbaugebiete gezogen worden. ${ }^{8}$ Seit Frühjahr 1997 wirkt das IRS am „Planspiel Leipzig-Griinau” mit. 
2 Die differenzierte räumliche Einordnung der Großsiedlungen als wesentliches Kriterium künftiger Entwicklungsperspektiven

Bereits seit den ersten komparativen Untersuchungen zum Bestand an Großsiedlungen in West und Ost wurde sehr eindrucksvoll auf einige prinzipielle Unterschiede hingewiesen: ${ }^{9}$

- Die Anzahl der Großwohnsiedlungen und die Anzahl der in ihnen bestehenden Wohnungseinheiten sind - trotz des bedeutend geringeren Bevölkerungsanteils - in den neuen Bundesländern erheblich größer als die in den alten Ländern.

- Die Großsiedlungen im Osten sind ihrem jeweiligen Umfang nach in der Regel bedeutend größer. $27 \mathrm{Ge}$ biete im Osten umfassen 10000 und mehr Wohnungen, im Westen hingegen nur sieben. Die zehn größten Großsiedlungen Deutschlands befinden sich ausschließlich in den neuen Ländern.

- Die in den neuen Ländern vorhandenen großen Neubaugebiete sind ihrer Entstehungszeit nach meist auch jünger. Ein sehr großer Teil entstand erst in den 70er und 80er Jahren im Zuge des Wohnungsbauprogramms der DDR, als in den Ländern der „alten” Bundesrepublik der Bau neuer Satellitenstädte und Großsiedlungen bereits ein ,auslaufendes Modell" geworden war.

Hinzu kommt aber auch ein wesentlicher Unterschied, der nach unseren Erfahrungen bisher zu Unrecht etwas im Hintergrund geblieben ist, aber für die Zukunftsperspektive der Großsiedlungen im Osten aller Wahrscheinlichkeit nach eine nicht zu unterschätzende Rolle spielen wird: die unterschiedliche räumliche Einordnung der Großsiedlungen.

Wie die 1996 von der Bundesforschungsanstalt für Landeskunde und Raumordnung herausgegebene Grafikübersicht ${ }^{10}$ der Großsiedlungen mit mehr als 1000 Wohnungen in der
Bundesrepublik Deutschland ausweist (Abb. 1), liegen die Standorte der westdeutschen Großsiedlungen nahezu ausnahmslos $(92 \%)$ in den jeweiligen Verdichtungsräumen der dominierenden Großstädte und Ballungsgebiete. ${ }^{11}$ In den neuen Ländern hingegen befindet sich der weitaus größte Teil der großen Neubaugebiete - nach unseren Ermittlungen $60 \%$ - außerhalb solcher Verdichtungsräume, d. h. in mittleren bzw. kleineren Städten. Berücksichtigt man die jeweilige Anzahl der Wohnungen je Großsiedlung, so reduziert sich dieser Anteil auf $40 \%$, ist aber immer noch unvergleichlich größer als der entsprechende Anteil in den alten Ländern (7\%).

Auf die Gründe, die zur Entstehung dieses erheblichen Unterschieds in der territorialen Einordnung der Großsiedlungen in Ost und West geführt haben, muß an dieser Stelle nicht gesondert eingegangen werden. Nur soviel sei gesagt: Die in Westdeutschland in den 50er und 60er Jahren meist am Rand der großen Städte, nicht selten auf den Flächen oder in unmittelbarer Nachbarschaft von Baracken oder Behelfsheimen zur quantitativen Wohnungsversorgung von Flüchtlingen, Obdachlosen oder anderen Angehörigen unterer Bevölkerungsschichten entstandenen Großsiedlungen wurden im Rahmen des öffentlich geförderten Mietwohnungsbaus errichtet. In den späten 60er und in den 70er Jahren entstanden solche Siedlungen als neue Stadtteile oder Trabantenstädte vor allem in den industriellen Ballungsgebieten zur Abdeckung des inzwischen gewachsenen qualitativen Wohnungsbedarfs sowie zum Auffangen der aus innerstädtischen Sanierungsgebieten verdrängten, wirtschaftlich schwachen Bevölkerung. ${ }^{12}$

In der ehemaligen DDR hingegen, wo bereits seit den frühen 50er Jahren auf der Grundlage eines zentralistischen Wirtschaftssystems territorialplanerisch eine von neuen Zielstellungen ausgehende „Standortverteilung der Produktivkräfte" angesteuert wurde, die darauf gerichtet war, historisch überkommene regionale Disparitäten z. B. zwischen den industriell geprägten südlichen Landesteilen und dem traditionell agrarisch geprägten Norden und Osten zu überwinden, war der massenhaft und industriell gefertigte Wohnungsbau in Form neuer Wohnkomplexe und -gebiete „auf der grünen Wiese" stets mit der gleichzeitig einhergehenden Errichtung neuer Produktionsbetriebe und Industriegebiete bzw. der Ansiedlung von Einrichtungen der Landesverteidigung oder ähnlichem verbunden. ${ }^{13}$

So sind sie denn in meist beachtlich kurzen Bauzeiträumen förmlich aus dem märkischen Streusandboden ,gestampft" worden: das Petrolchemische Kombinat und die zahlreichen Neubaukomplexe in Schwedt/Oder, das Textilkombinat und die Großsiedlung Obersprucke in Guben, das Stahl- und Walzwerk Brandenburg und die Wohngebiete Görden und Hohenstükken, das Frankfurter Halbleiterwerk und das Neubaugebiet Neuberesinchen, das Automobilwerk Ludwigsfelde und die Potsdamer Straße, das Fleischverarbeitungskombinat in Eberswalde und das heutige Brandenburgische Viertel. Zahlreiche ähnliche Beispiele gibt es in den anderen neuen Bundesländern.

Viele der bis zur Industrieansiedlung wirtschaftlich relativ bedeutungslosen Mittel- und Kleinstädte haben auf diese Weise in vergleichsweise wenigen Jahren einen außerordentlich starken Einwohnerzuwachs erfahren. Der Anteil der Wohnungen in Großwohnsiedlungen beträgt in solchen schnell gewachsenen mittleren Industriestädten wie die genannten in Brandenburg bzw. in anderen Regionen, z. B. Leinefelde, Weißwasser und Hoyerswerda, nicht selten über 60 oder $70 \%$ (Abb. 2). Das praktische Fehlen eines neuen Wohneigentumssektors in der DDR - abgesehen von dem relativ geringen Anteil des Eigenheimbaus seit den 70er Jahren - hat dabei insbesondere in den mittleren und kleineren Städten zu einem sehr undifferenzierten Wohnungsangebot, zu einem Mangel an unterschiedlichen Wohnformen und -qualitäten und vielfach auch zu 


\section{Abbildung 1}

Großsiedlungen mit mehr als 1000 Wohnungen in der Bundesrepublik Deutschland

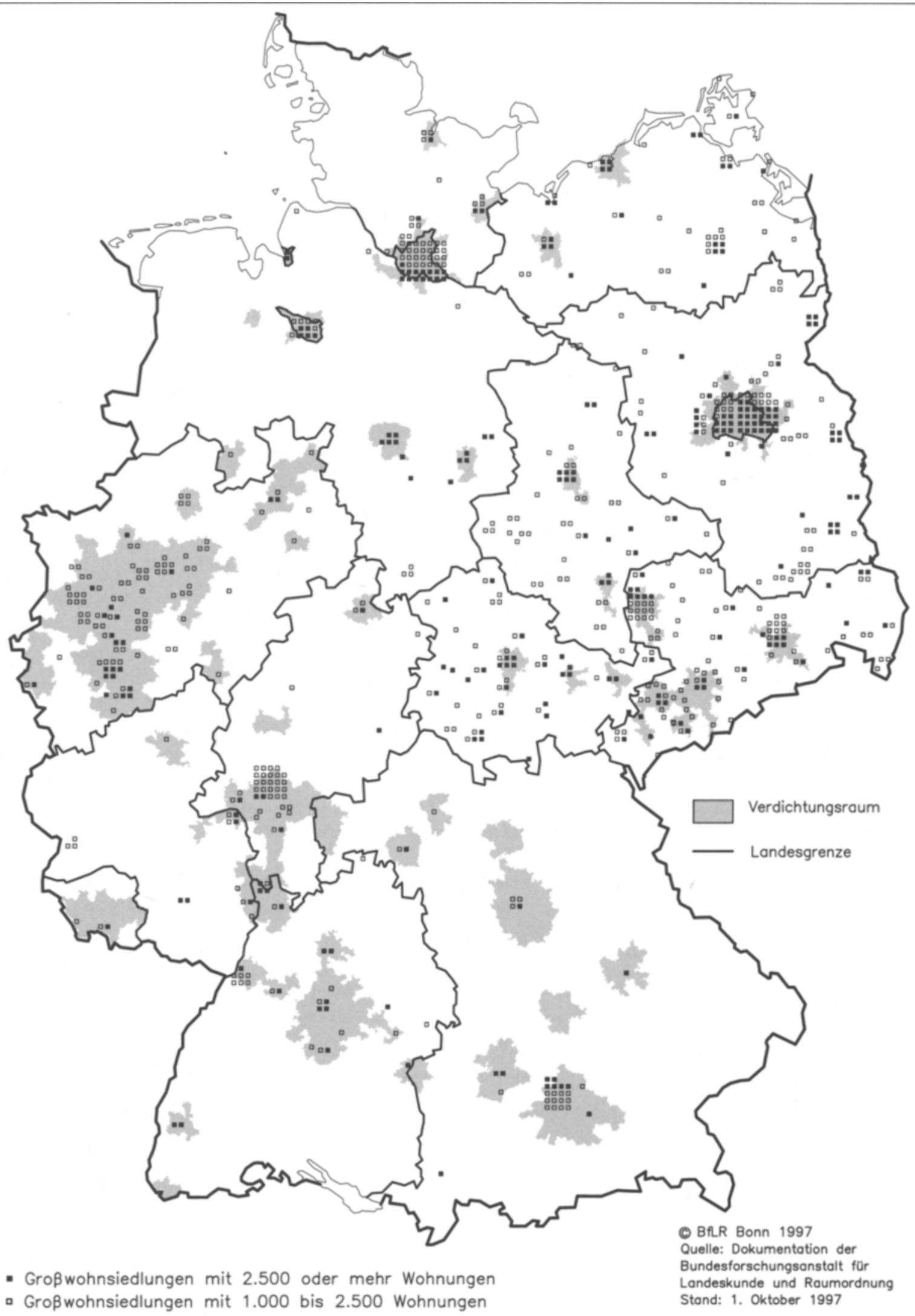




\section{Abbildung 2}

Anteil der Wohnungen in Großsiedlungen am Wohnungsbestand in Städten unterschiedlicher Größengruppen

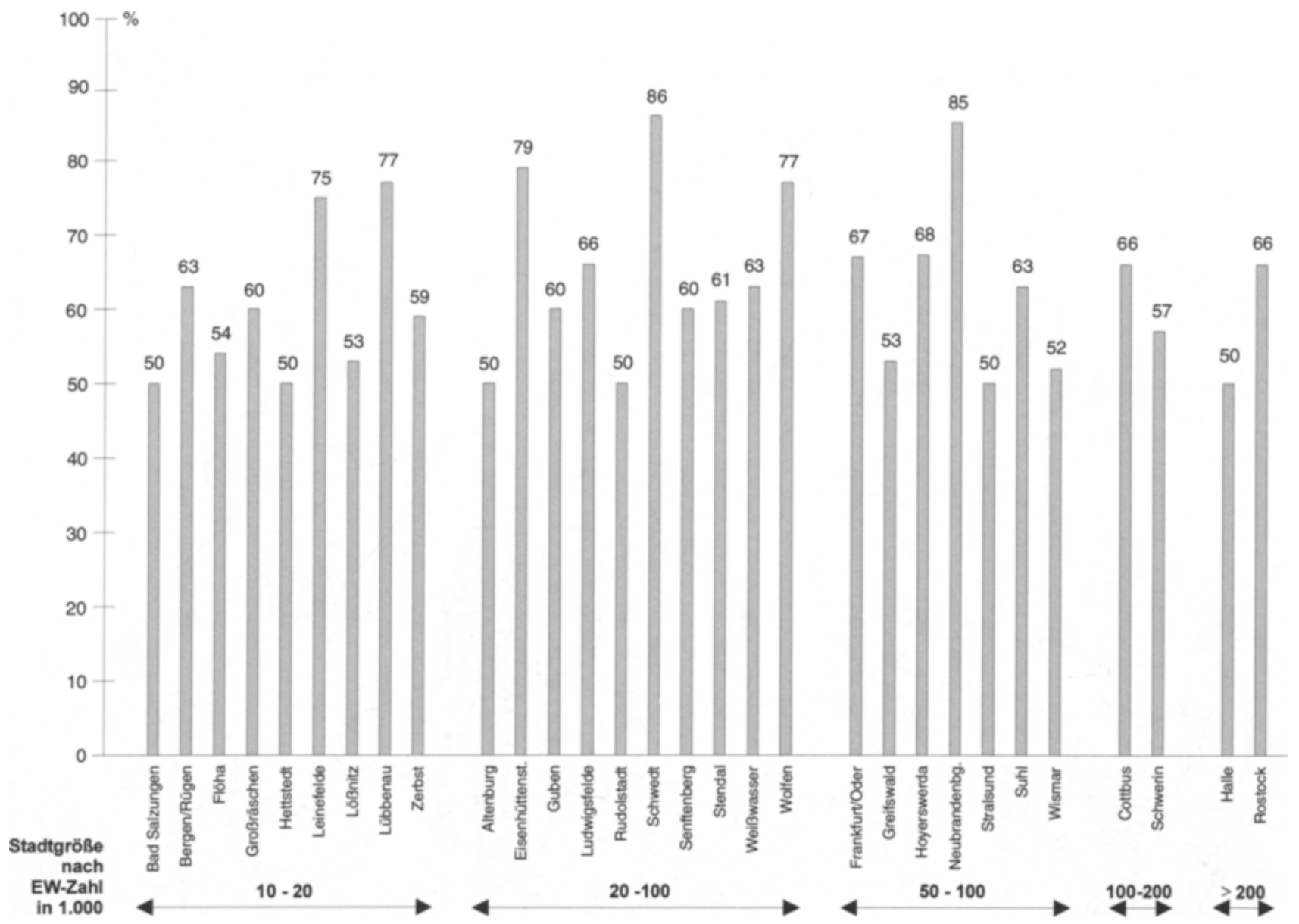

sehr ungünstigen räumlich-gestalterischen Beziehungen zwischen den neu entstandenen Wohnkomplexen und den historisch gewachsenen Stadtgebieten geführt.

Für den heutigen Umgang mit solchen Großsiedlungen in mittleren und kleineren Städten und für ihre mittel- und langfristige Perspektive sind damit zumindest drei Bedingungen gegeben, die im Vergleich zu gleichzeitig entstandenen Großwohnsiedlungen in Ostberlin oder anderen Verdichtungsräumen der neuen Bundesländer von vornherein prinzipiell als ungünstiger zu betrachten sind:

1. die aus der Entstehungszeit herrührende, bisher vorrangige Bindung an frühere Industriegroßbetriebe oder sonstige Arbeitsstätten, die inzwischen „abgewickelt" wurden bzw. nur noch über vergleichsweise wenige Arbeitsplätze verfügen,
2. die, bezogen auf die jeweilige Gesamtstadt, ausgeprägte Einseitigkeit des Wohnungsangebots in Gestalt hochtypisierter, industriell gefertigter Gebäudeserien, die heute nur noch teilweise dem veränderten Wohnungsbedarf in der jeweiligen Stadt entsprechen,

3. die vielfach sehr unbefriedigende städtebauliche Einordnung dieser Baukomplexe in wertvolle, sensible Landschaftsräume und historisch entstandene Stadtstrukturen.

Bezugnehmend auf die drei Kriterien

- Wirtschaftsentwicklung im Raum,

- Anteil der Großsiedlungen am Wohnungsbestand einer Stadt,

- städtebauliche und sozialkulturelle Integration der Großsiedlungen,

lassen sich folgende Zusammenhänge erkennen:Je einseitiger sich eine Großwohnsiedlung an eine monostrukturel- le Wirtschaftsentwicklung koppelte, je größer der Anteil der Wohnungen in den Großsiedlungen einer Stadt am gesamten Wohnungsbestand der betreffenden Stadt ist und je geringer die städtebauliche und sozial-strukturelle Integration der Großsiedlungen ausgebildet ist, desto komplizierter und schwieriger wird voraussichtlich ihre mittel- und langfristige Entwicklungsperspektive sein.

Demnach muß man sich um die $\mathrm{Zu}$ kunft der Großsiedlungen in wirtschaftlich prosperierenden oder wenigstens im großen und ganzen relativ stabilen Mittel- und Kleinstädten, z. B. in der Industriestadt Ludwigsfelde innerhalb des sogenannten engeren Verflechtungsraumes Brandenburg-Berlin oder in Mittweida und in Lutherstadt Wittenberg, vermutlich in den nächsten Jahren weniger Sorgen machen müssen als beispielsweise um Groß- 
siedlungen in abgelegenen, inzwischen weitgehend deindustrialisierten Regionen wie Leinefelde (Kreis Eichsfeld), Sangerhausen, Wolfen (Kreis Bitterfeld) sowie in solchen fruheren Industrieschwerpunkten wie Hoyerswerda, Lübbenau und Weißwasser in der Lausitz oder in Städten wie Schwedt/Oder, Guben und Forst in der Oder-Neiße-Grenzregion zu Polen.

Im Abschlußbericht zum ExWoStForschungsfeld „Städtebauliche Entwicklung großer Neubaugebiete in den neuen Bundesländern und BerlinOst" haben die BfLR und das Stadtbüro Hunger bereits grundsätzlich auf diese Problematik hingewiesen, wenn sie feststellen, daß die Perspektiven großer Neubaugebiete um so günstiger sind, ,je besser die wirtschaftliche und soziale Perspektive der Region bzw. der betreffenden Stadt ist"..14

Und Hartmut Häußermann, Sozialwissenschaftler an der Humboldt-Universität Berlin, unterscheidet hinsichtlich der verschiedenen Entwicklungsrichtungen für die ostdeutschen Neubaugebiete die folgenden drei „Typen“:

„- Großsiedlungen, die integraler Bestandteil einer Stadt werden und komfortable/bezahlbare Wohnungen und Wohnmilieus für die untere Mittelschicht bieten; Beispiele sind Berlin-Marzahn, Potsdam-Am Stern.

- Großsiedlungen, die nicht in das Bau- und Funktionsgefüge einer Stadt oder eines Gebietes integriert werden können und früher oder später rückgebaut werden (müssen). Dazu zählen die Großsiedlungen in Mittelstädten, die zu DDRZeiten als 'Werkssiedlung' für einzelne Großbetriebe errichtet wurden, die nun stillgelegt sind. Guben-Obersprucke, Schwedt/ Oder oder Wolfen-Nord sind Beispiele für diesen Typus.

- Großsiedlungen, die aus wohnungspolitischen Gründen zur Unterbringung von 'sozialen Problemgruppen', wie Sozialhilfeempfänger, Aussiedler usw. dienen. $\mathrm{Zu}$ dieser Gruppe werden vor allem die Gebiete gehören, die ge- genüber der eigentlichen Stadt eine extreme Randlage haben. Erste Trends in Richtungen einer solchen sozialen Marginalisierung deuten sich in einigen Großsiedlungen an. “15

Hinweise auf eine direkte Raumrelevanz der Chancen der Großsiedlungen sind in diesen Worten zwar nicht enthalten, lassen sich aber mit ihnen in Verbindung bringen. Hingegen bleiben die in einer Studie des Eduard Pestel Instituts für Systemforschung e. V. Hannover $^{16}$ enthaltenden Schlußfolgerungen zum voraussichtlichen Rückbaupotential des Plattenwohnungsbestandes in den neuen Bundesländern lediglich bei einer Differenzierung nach ländlichen Räumen, Mittel- und Oberzentren, Ostberlin sowie einzelnen Geschoßbauten in Stadtlagen stehen, ohne daß weitergehende Bezüge zur wirtschaftlichen Struktur der jeweiligen Räume angesprochen werden.

\section{Erste Anzeichen struktureller Gefährdungen der ostdeutschen Großsiedlungen}

In allen offiziellen Materialien zur Problematik der Großwohnsiedlungen, insbesondere in Ostdeutschland, wird mit großer Übereinstimmung festgestellt, daß diese Siedlungen für die Wohnungsversorgung unverzichtbar sind. ${ }^{17}$

Diese Grundposition war und ist Ausgangsbasis für die seit nunmehr bereits über sechs Jahre laufenden Bemühungen um umfangreiche Instandsetzungs- und Sanierungsmaßnahmen in diesen Gebieten sowie vor allem auch um eine sozialorientierte Wohnumfeldverbesserung. Die bisherigen Ergebnisse, z. B. in den Ostberliner Neubaustadtteilen Marzahn, Hellersdorf und Hohenschönhausen, in Cottbus und Gera, Mittweida und Güstrow sind in vielfacher Weise anregend und beispielhaft auch für ähnliche Herausforderungen in Großwohnsiedlungen der mittel- und osteuropäischen Länder.
Dennoch darf nicht uibersehen werden, $\mathrm{daB}$ insbesondere in den letzten zwei, drei Jahren in einer ganzen Reihe ostdeutscher Großsiedlungen Fluktuation und Migration erheblich zugenommen haben und mitunter bereits auch dauerhafter Wohnungsleerstand in Plattenneubaugebieten in bedenklichem Maße auftritt.

Wurde im Abschlußbericht zum ExWoSt-Forschungsfeld 1995 noch festgestellt, daß sich das Umzugsgeschehen in den neuen Ländern von ca. 7 \% 1990 auf ca. $4 \% 1993$ reduziert hat, so sind inzwischen Fluktuationsraten von 7 bis $14 \%$ jährlich in vielen Gebieten keine Ausnahmen mehr. ${ }^{18}$ Die Zielpunkte der Umzüge liegen dabei teils innerhalb der Großsiedlungen selbst, teils in anderen ähnlich strukturierten Stadtteilen, teils aber auch in neuen, geringgeschossigen Wohnanlagen am Stadtrand sowie - in vergleichsweise geringerem Umfang - in sanierten Innenstadtgebieten.

Die potentielle Migrationsbereitschaft der Bewohner von Plattenbausiedlungen, d. h. die konkreten bzw. wahrscheinlichen Auszugsabsichten innerhalb eines Zeitraums von ca. zwei Jahren, wurden in jüngster Zeit für unterschiedliche Großwohnsiedlungen mit ca. 25 bis $40 \%$ ermittelt, z. B. Rostock Groß Klein $24 \%{ }^{19}$, Berlin-Marzahn $32 \%$, Leinefelde $32 \%$, Dresden-Gorbitz $34 \%$ und Leipzig-Grünau $40 \%$. Wie entsprechende sozialwissenschaftliche Untersuchungen ausweisen, sind die Wegzugsabsichten am deutlichsten ausgeprägt bei:

- Haushalten mit einem höheren Einkommen,

- Bewohnern mit Hoch- und FachschulabschluB,

- jüngeren Altersgruppen.

Hohe Fluktuationsraten und stark ausgeprägte Migrationsbereitschaft müssen freilich nicht ,,automatisch" zu hohen Wohnungsleerständen führen, sondern sind zunächst einmal Ausdruck der seit der Wende in Ostdeutschland einsetzenden, auf der Entwicklung eines Wohnungsmarktes gegründeten Ausdifferenzierungs- 
bzw. Segregationsprozesse-Vorgänge, die sich im Prinzip in allen Regionen vollziehen, wenn auch in differenzierter Form und in unterschiedlichem Tempo.

Wie Abbildung 3 zeigt, treten nach unseren Ermittlungen überdurchschnittliche Leerstandszahlen gegenwärtig nahezu ausschließlich in peripheren, strukturgeschwächten Räumen, z. B.

\section{Abbildung 3}

Großsiedlungen mit ausgeprägtem Wohnungsleerstand in Relevanz zu den Verdichtungsräumen in den neuen Ländern

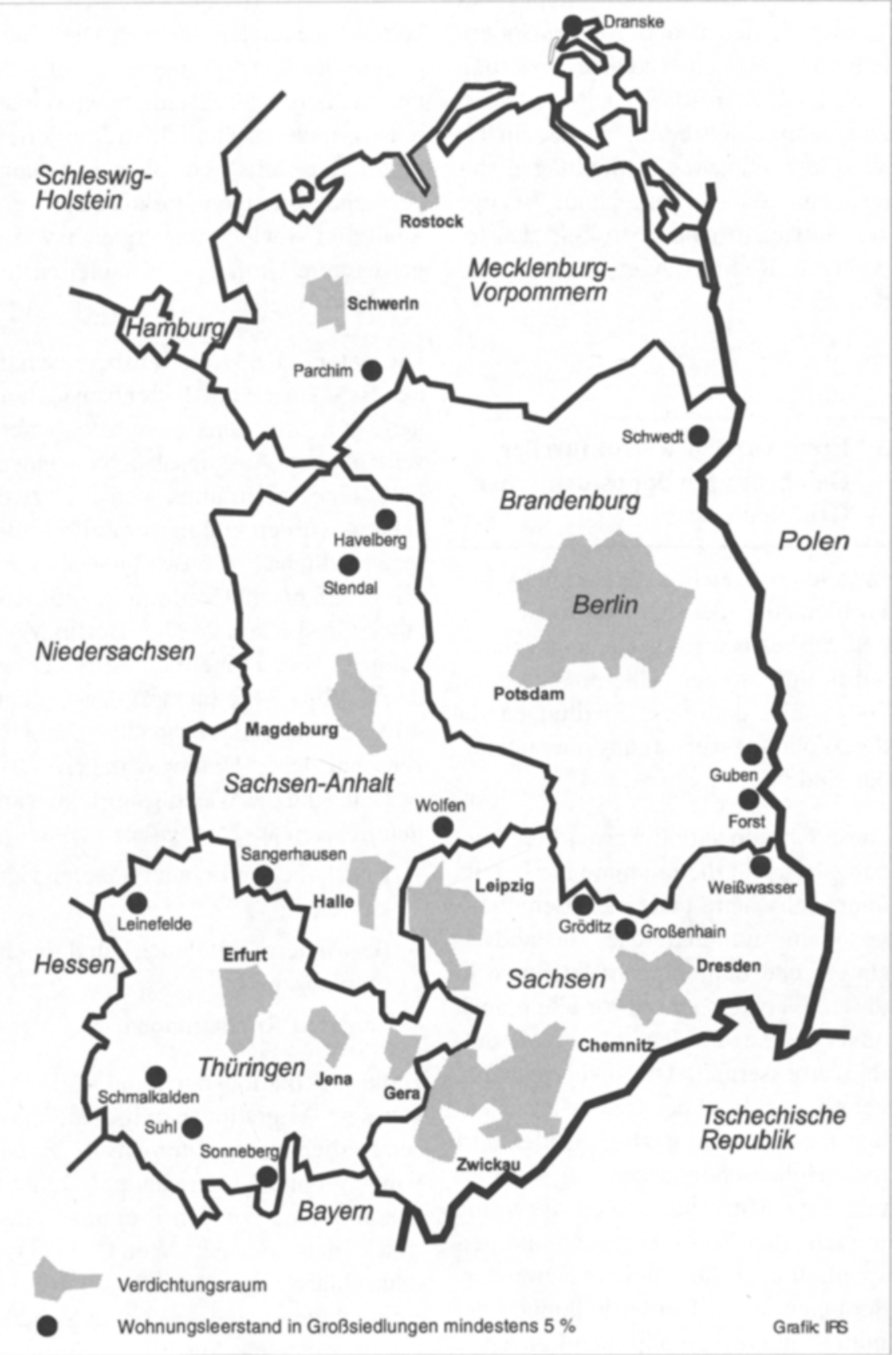

zum Teilrückbau (Hoyerswerda) sowie Abriß (Guben) einzelner Wohngebäude geführt.

Auf der anderen Seite gibt es in den Ostberliner Großwohnsiedlungen, in Leipzig-Grünau oder Dresden derzeit und in absehbarer Zukunft keinen erkennbaren Trend zu einer besonderen Leerstandsentwicklung. Vielmehr finden sich z. B. in Berlin-Marzahn, -Hellersdorf und -Altglienicke bemerkenswerte Beispiele für eine Erweiterung des Wohnungsbestands der Großwohnsiedlungen durch ein- oder angelagerten ergänzenden Wohnungsneubau. ${ }^{23}$ Ähnliches vollzieht sich inzwischen auch in Ludwigsfelde ${ }^{24}$, Rostock-Nordost und Gera-Lusan, d. $h$. vorwiegend in Räumen, die als Verdichtungsräume ein breiteres Spektrum an Arbeitsplätzen bieten.

\section{Sieben Punkte zu künftigen Großsiedlungsstrategien}

Damit die ostdeutschen Großwohnsiedlungen der Gefahr entgehen, zu „sozialen Brennpunkten” zu werden, sondern statt dessen die Chance erhalten, sich zu eigenständigen, integrierten und vielfältigen Stadtteilen zu entwickeln (Abb. 4), sind unserer Meinung nach vor allem die folgenden sieben Punkte zu künftigen Großsiedlungsstrategien von besonderer $\mathrm{Be}$ deutung.

(1)

Es geht nicht um universale Strategien, die für alle Großsiedlungen gleichermaßen gelten, sondern um lokal und regional differenzierte Strategien, die die spezifischen Gegebenheiten und Rahmenbedingungen berücksichtigen.

(2)

Uns helfen keine „aus sich selbst” entwickelten, introvertierten Großsiedlungskonzepte, sondern nur aus gesamtstädtischen bzw. übergreifenden wirtschaftlichen, sozial-kulturellen und ökologischen Kontexten abgeleitete Strategien.

(3)

Erforderlich sind Strategien mit gestaffelten Zeithorizonten (kurz-, mit- 
Abbildung 4

Grundlegende Alternativen für die Perspektive

der ostdeutschen Großwohnsiedlungen

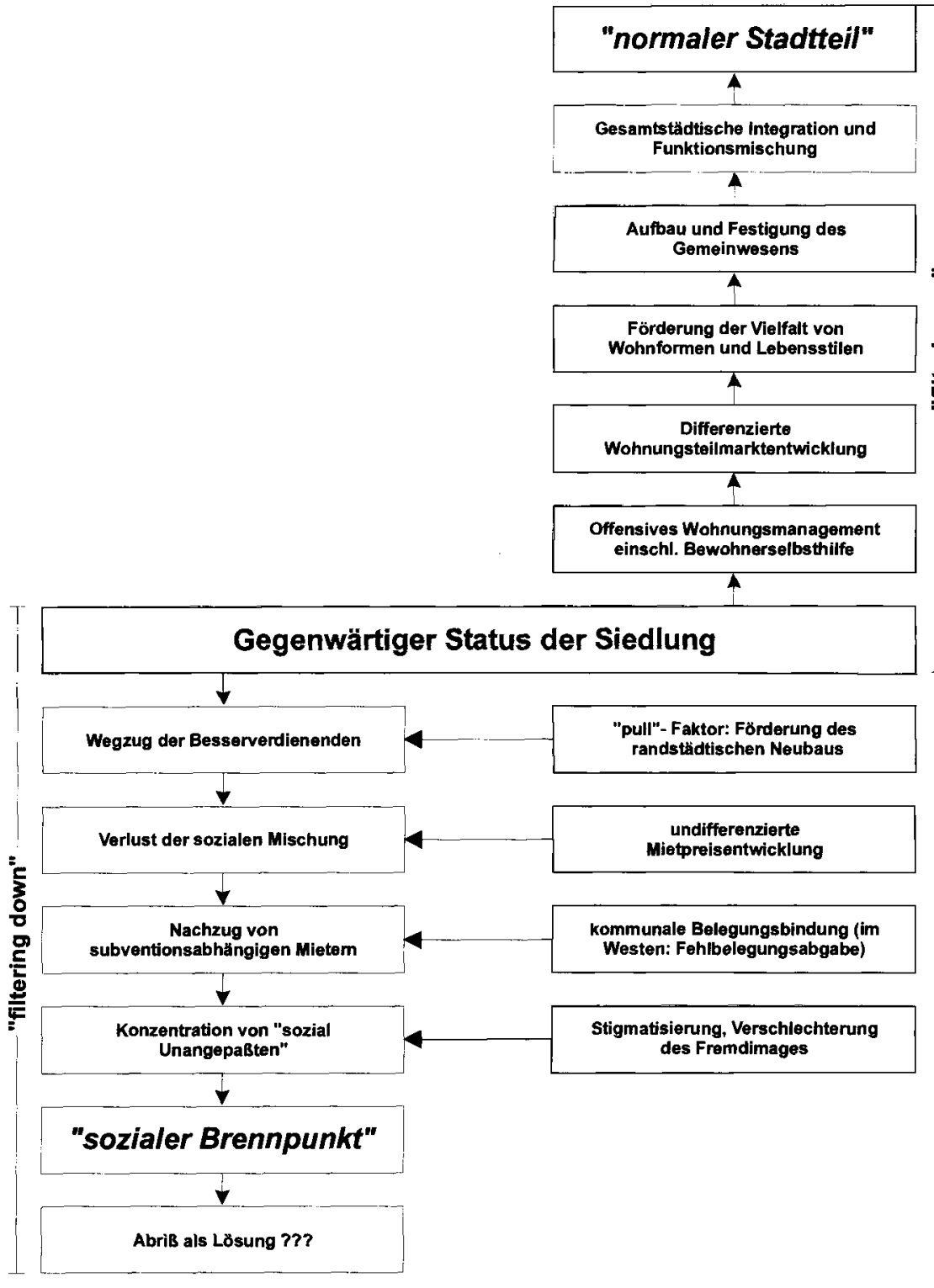

(7)

Ziele künftiger Strategien sollten eigenständige, integrierte Stadtteile mit vielfältigen Funktionen, differenzierten Standards, Wohnmilieus und Lebensstilen, neuen Formen des Stadtteil- und Wohnungsmanagements einschließlich gegebenenfalls innovativer und unkonventioneller Verwendung von leerstehender oder untergenutzter Bausubstanz sein.

\section{Ein neues Modell für öffentliche Interventionen in städtische Problemgebiete - Erfahrungen und Anregungen aus Frankreich $^{28}$}

Langjährige Erfahrungen mit der Rehabilitation der in der 60er und 70er Jahren in Frankreich entstandenen suburbanen „Grands Ensembles” haben sehr deutlich gezeigt, daß die sozialen Probleme in solchen ,heruntergekommenen Stadtvierteln" mit städtebaulichen Mitteln allein nicht zu lösen sind.

In diesen den ostdeutschen Neubaugebieten auf den ersten Blick oft durchaus sehr ähnlichen großen randstädtischen Siedlungen mit ihrem hohen Anteil vielgeschossiger und Hochhausbebauung, einer meist eintönigen, aus der industriellen Fertigung hervorgegangenen Gestalt und einer einseitig auf das bloße Wohnen beschränkten Funktion entwickeln sich seit den 80er Jahren handfeste sozialökonomische und sozialkulturelle Probleme: eine weit über dem Durchschnitt liegende Arbeitslosigkeit, ein vergleichsweise geringes Ausbildungsniveau, eine groBe Anzahl von Schulabbrechern, hoher Anteil Drogensüchtiger und wachsende Kriminalität, insbesondere unter Jugendlichen. Dabei muß man berücksichtigen, daß sich diese Probleme vor allem auf die dort vorherrschenden Bevölkerungsschichten der Einwanderer aus Nordafrika sowie anderer ,,ausgegrenzter" sozialer Gruppen konzentrieren.

Der französische Staat ist seit Mitte der 70er Jahre darum bemüht, die Zustän- 
de in solchen Gebieten durch eine Kombination unterschiedlicher Maßnahmen zu verbessern, und entwickelte dazu diverse Rehabilitationssprogramme wie z. B. „Wohnen und soziales Leben”, „Vom Wohngebiet zur Stadtwerdung”, „Soziales-urbanes Entwicklungsprogramm". Kernpunkt besonders der jüngeren Programme sind dabei sog. Stadtverträge, die für eine Laufzeit von fünf Jahren zwischen dem Staat und der Kommune geschlossen werden und vielfältige soziale Maßnahmen wie z.B. zur Berufsausbildung, zur Arbeitsplatzbeschaffung, zur Unterstützung der lokalen Ökonomie u. a. umfassen. Bis heute wurden 214 Stadtverträge für insgesamt über 1300 Wohngebiete abgeschlossen. Im Jahre 1994 kam es zusätzlich zur Einführung von „GroBen Stadtprojekten" in zwölf Gebieten, die durch ihre räumlichen Dimensionen und die Dringlichkeit ihrer sozialen Probleme besonders auffielen. Das Programm der „Großen Stadtprojekte" versucht in enger Partnerschaft mit dem privaten Sektor einen tiefen positiven Wandel in der sozialen, ökonomischen und kulturellen Entwicklung der Gebiete herbeizuführen. Die Regierung beteiligt sich seit 1996 unter anderem dadurch, daß sie auf der Grundlage des „Vertrages zur Wiederbelebung" (Pacte de relance) in 700 sog. „sensiblen Zonen der Städte” insgesamt 100000 sozial-wirtschaftliche Arbeitsplätze mit der Hälfte der Kosten mitfinanziert - die andere Hälfte wird im Rahmen von öffentlichen Partnerschaften durch die Städte bzw. den staatlichen Wirtschaftssektor übernommen.

Auch wenn diese derzeit in Frankreich praktizierte neue „Politik für die Städte" nicht ohne weiteres ein Modell für die Beherrschung eventueller künftiger Problemeskalationen bei den in strukturschwachen Räumen gelegenen Großsiedlungen in den neuen Bundesländern sein kann, so sind ihre Ansätze dennoch für die neuen Länder ohne Zweifel sehr interessant, da sie

a) - wie bereits festgestellt - über bauliche und städtebauliche Frage- stellungen von Anfang an hinausgehen,

b) stets soziale und ökonomische Probleme integriert aufgreifen und

c) jeweils differenziert auf die spezifischen Situationen in den betreffenden Regionen und Kommunen eingehen.

In einer künftigen Forschungskooperation mit dem Institut d' Aménagement et d' Urbanisme de la Région d' Ile de France will das IRS diesen wichtigen Ansätzen weiter ,auf der Spur" bleiben.

\section{Anmerkungen}

(1)

Ermittlungen der Bundesforschungsanstalt für Landeskunde und Raumordnung 1995 auf der Grundlage der vom Institut für Regionalentwicklung und Strukturplanung 1994 aufgestellten Standortübersicht

(2)

Lautenschläger, Rolf: Gegenwelten der Zivilisation. Die Plattenbau-Ghettos an den Rändern der Metropolen sind städtebauliche Sozialfälle: monofunktional, ungeliebt und verwahrlost. In: taz, 11.3.1997

(3)

Berg, Stefan: Ein Ort - nicht zum Leben. Schwedt, die östlichste Chemiestadt Deutschlands. In: Der Spiegel (1996) 47, S. 96-110

(4)

Bundesministerium für Raumordnung, Bauwesen und Städtebau (Hrsg.): Vitalisierung von Großsiedlungen. Expertise: Informationsgrundlagen zum Forschungsthema Städtebauliche Entwicklung von Neubausiedlungen in den fünf neuen Bundesländern. - Bonn 1991

\section{(5)}

Bundesforschungsanstalt für Landeskunde und Raumordnung (Hrsg.): ExWoSt-Informationen zum Forschungsfeld Entwicklung großer Neubaugebiete, Heft 1 (7/1991), Heft 2 (8/1992), Heft $3(8 / 1993)$

(6)

Liebmann, Heike: Siedlungsporträt Ludwigsfelde - Großes Neubaugebiet in einer prosperierenden Mittelstadt. In: Bundesministerium für Raumordnung, Bauwesen und Städtebau (Hrsg.): Internationales Forschungsseminar Große Neubaugebiete. - Bonn 1993, S. 46-51

\section{(7)}

Bundesministerium für Raumordnung, Bauwesen und Städtebau (Hrsg.): Bund-Länder-Förderprogramm Städtebauliche Weiterentwicklung großer Neubaugebiete in den neuen Ländern und im Ostteil Berlins. Ziele und erste Er- gebnisse. Institut für Regionalentwicklung und Strukturplanung. - Bonn, Berlin 1996

(8)

Bundesministerium für Raumordnung, Bauwesen und Städtebau, Sächsisches Staatsministerium des Innern, Amt für Stadtsanierung und Wohnungsbauförderung Leipzig (Hrsg.): Fachveranstaltungen zur städtebaulichen Weiterentwicklung großer Neubaugebiete anläßlich des 20jährigen Bestehens des Stadtteils LeipzigGrünau. Dokumentation. - Bonn, Berlin 1997

(9)

Rietdorf, Werner: Neubauwohngebiete in den neuen Bundesländern, Bestandsaufnahme und Zukunftsperspektive. = Architektur, Berlin (1991) 9; Bundesministerium für Raumordnung, Bauwesen und Städtebau (Hrsg.): Großsiedlungsbericht 1994. - Bonn 1994. = Drucksache 12/8406; Funtich, Manfred; Manner, Harald: Großwohnsiedlungen - gestern, heute, morgen. In: Bundesforschungsanstalt für Landeskunde und Raumordnung (Hrsg.): Große Neubaugebiete - Bestand, städtebauliche Handlungsfelder und Perspektiven. In: Informationen zur Raumentwicklung, Bonn (1994), H. 9 S. 567-585

(10)

Bundesministerium für Raumordnung, Bauwesen und Städtebau (Hrsg.): Städtebauliche Weiterentwicklung von Großwohnsiedlungen in der Bundesrepublik Deutschland. - Bonn, Berlin 1996 (Faltblatt)

(I1)

Verdichtungsräume gemäß den Beschlüssen des Hauptausschusses der Ministerkonferenz für Raumordnung v. 21.11.1968 und 17.9.1993

(12)

Bundesministerium für Raumordnung, Bauwesen und Städtebau (Hrsg.): Städtebaulicher Bericht - Neubausiedlungen der 60er und 70er Jahre. Probleme und Lösungswege. - Bonn 1988

(13)

Hunger, Bernd: Die Bedeutung großer Neubau gebiete in der Wohnungs- und Städtebaupolitik der DDR - historischer Rückblick. In: Bundes forschungsanstalt für Landeskunde und Raumordnung (Hrsg.): Große Neubaugebiete - Bestand, städtebauliche Handlungsfelder und Perspektiven. In: Informationen zur Raumentwicklung, Bonn (1994), H. 9, S. 595-602

(14)

Stadtbüro Hunger; Bundesforschungsanstalt für Landeskunde und Raumordnung (Hrsg.): Experimenteller Wohnungs- und Städtebau: Forschungsfeld „Städtebauliche Entwicklung groBer Neubaugebiete in den neuen Bundesländern und Berlin-Ost". Endbericht. - Berlin, Bonn 1996, S. 83

\section{(15)}

Häußermann, Hartmut: Die Transformation des Wohnungswesen. In: Strubelt, Wendelin; Genosko; Bertram u.a.: Städte und Regionen - Räumliche Folgen des Transformationsprozesses. Berichte zum sozialen und politischen Wandel in Ostdeutschland. - Opladen 1996, S. 309 
(16)

Pestel Institut für Systemforschung e.V. Hannover (Hrsg.): Zwischen Sanierung und Abriß. Plattenbauten in den neuen Bundesländern. Leipzig 1996

(17)

Siehe z.B. den unter Anm. (9) genannten GroB siedlungsbericht 1994, den Zwischenbericht zum Bund-Länder-Förderungsprogramm [Anm. (7)], das Faltblatt von 1996 [Anm. (10)] und die Tagungsdokumentation Leipzig-Grünau 1996 [Anm. (8)]

(18)

Rietdorf, Werner: Stand und Perspektive der Strategiediskussion zur Weiterentwicklung ostdeutscher Großsiedlungen. Vortrag auf dem Wissenschaftlichen Symposium „Perspektiven der großen Neubaugebiete in den neuen Bundesländern" am 21.4.1997 im Institut für Regionalentwicklung und Strukturplanung (IRS) in Erkner

(19)

Hohn, Uta: Rostock Groß Klein - Transformationsprozesse in einer ostdeutschen Großsiedlung (1992-1995). 50. Deutscher Geographentag Potsdam 1995, Bd. 2

(20)

Wohnungsbaugesellschaft Marzahn $\mathrm{mbH}$ (Hrsg.): Wir in Marzahn. Mieterbarometer 1996. Fakten, Meinungen, Tendenzen. - Berlin 1996

(21)

Kahl, Alice: Leipzig-Grünau - Ergebnisse und Ableitungen aus der soziologischen Intervallstudie (1979 bis 1995) zum Wohnen in der Großsiedlung. In: Rietdorf, Werner (Hrsg.): Weiter wohnen in der Platte. Probleme der Weiterentwicklung großer Neubauwohngebiete in den neuen Bundesländern. - Berlin 1997

(22)

Leerstand in Schwedt ca. 9,8 \% des Wohnungsbestandes insgesamt (Konzentration in den Wohngebieten Am Waldrand etwa $16 \%$ und Talsand ca. $14 \%$ Leerstand); im Gubener Neubaugebiet Obersprucke ca. $12 \%$, im Bestand der städtischen Wohnungsgesellschaft Hoyerswerda ca. 14 Prozent (Transferveranstaltung des IRS „Konzepte für den Umgang mit Wohnungsleerstand am 30.10.1997 in Schwedt/ Oder)

(23)

Schümer-Strucksberg, Monica: Fünf Jahre Berliner Großsiedlungsstrategie - Bilanz und Ausblick. In: Rietdorf, Werner (Hrsg.): Weiter wohnen in der Platte. Probleme der Weiterentwicklung großer Neubauwohngebiete in den neuen Bundesländern. - Berlin 1997

(24)

Liebmann, Heike: Modellfall Ludwigsfelde. In: Rietdorf, Werner (Hrsg.): Weiter wohnen in der Platte. Probleme der Weiterentwicklung großer Neubauwohngebiete in den neuen Bundesländern. - Berlin 1997
(25)

Rietdorf, Werner; Kühn, Manfred: Erhaltung und Vitalisierung von Großsiedlungen als ein Gebot nachhaltiger Stadtentwicklung. In: Rietdorf, Werner (Hrsg.): Weiter wohnen in der Platte. Probleme der Weiterentwicklung großer Neubauwohngebiete in den neuen Bundesländern. Berlin 1997; vgl. auch die folgenden Publikationen: Bundesforschungsanstalt für Landeskunde und Raumordnung (Hrsg.): Städtebaulicher Bericht Nachhaltige Stadtentwicklung - Herausforderungen an einen ressourcenschonenden und umweltverträglichen Städtebau. - Bonn 1996, S. 39-42; Bundesministerium für Raumordnung, Bauwesen und Städtebau (Hrsg.): Siedlungsentwicklung und Siedlungspolitik. Nationalbericht Deutschland. - Bonn 1996, S. 64; dass. (Hrsg.): Habitat II. Globale Best Practices - Initiative zur Verbesserung der Lebensumwelt. Deutsche Best Practices. Bonn 1996.

(26)

Großhans, Hartmut: Humanisierung der großen Siedlungen. Herausforderung, Ansätze und Leistungsbeiträge der gemeinnützig orientierten unternehmerischen Wohnungswirtschaft in den neuen Bundesländern. GdW Bundesverband deutscher Wohnungsunternehmen e.V. $=\mathrm{GdW}$ Informationen 50. - Köln 1997

(27)

Beer, Ingeborg: Bürgerbeteiligung in Plattenbausiedlungen - Modelle und Strategien für eine neue Urbanität? In: Rietdorf, Werner (Hrsg.): Weiter wohnen in der Platte. Probleme der Weiterentwicklung großer Neubauwohngebiete in den neuen Bundesländern. - Berlin 1997

(28)

Die nachstehenden Ausführungen gründen sich auf einen Vortrag, den Christine Lelévrier vom Institut d' Aménagement et d' Urbanisme de la Région d' Ile de France (Institut für Regionalplanung und Urbanisierung der Region Ile de France) am 21,4.1997 auf dem Wissenschaftlichen Symposium des IRS zum Thema „Perspektiven der großen Neubaugebiete in den neuen Bundesländern" gehalten hat. Unmittelbare Praxiseindrücke aus der Region Ile de France erhielten Mitarbeiter(innen) des IRS, darunter Werner Rietdorf und Heike Liebmann, im Januar 1997 auf einer entsprechenden Expertenreise (vgl. IRS aktuell 15/1997, S. 14/15).

Prof. Dr. Werner Rietdorf

Dipl-Ing. Heike Liebmann

Institut für Regionalentwicklung

und Strukturplanung e.V. (IRS)

Flakenstraße 28-31

15537 Erkner bei Berlin 